\title{
The relationship of neutrophil-to-lymphocyte ratio and platelet-to-lymphocyte ratio with bone mineral density in adolescent girls suffering from anorexia nervosa
}

\author{
Małgorzata Morawiecka-Pietrzak@1, 2, Żaneta Malczyk ${ }^{\circledR 1}$, Ewelina Dąbrowska ${ }^{3}$, Małgorzata Blaska ${ }^{3}$, \\ Mikołaj Pietrzak ${ }^{\circledR 4}$, Aleksandra Gliwińska ${ }^{\circledR 5}$, Anna Góra ${ }^{\circledR 1}$, Katarzyna Ziora ${ }^{\circledR 1}$, \\ Wojciech Pluskiewicz ${ }^{\circledR 6}$, Zofia Ostrowska ${ }^{\circledR 2}$ \\ ${ }^{1}$ Department of Paediatrics, Faculty of Medical Sciences in Zabrze, Medical University of Silesia, Katowice, Poland \\ ${ }^{2}$ Department of Medical and Molecular Biology, Faculty of Medical Sciences in Zabrze, Medical University of Silesia, Katowice, \\ Poland \\ ${ }^{3}$ Pediatric Endocrinology Ward, Independent Public Clinical Hospital No. 1 in Zabrze, Medical University of Silesia in Katowice, \\ Poland \\ ${ }^{4}$ Department of Internal Medicine, Angiology and Physical Medicine, Faculty of Medical Sciences in Zabrze, Medical University \\ of Silesia, Katowice, Poland \\ ${ }^{5}$ Pediatric Nephrology Ward with Dialysis Division for Children, Independent Public Clinical Hospital No. 1 in Zabrze, \\ Medical University of Silesia in Katowice, Poland \\ ${ }^{6}$ Department and Clinic of Internal Diseases, Diabetology and Nephrology, Metabolic Bone Diseases Unit, Faculty of Medical \\ Sciences in Zabrze, Medical University of Silesia in Katowice, Poland
}

\begin{abstract}
Introduction: Inflammation is supposed to be one of the factors contributing to decreased bone mineral density (BMD) in anorexia nervosa (AN). The neutrophil-to-lymphocyte ratio (NLR) and platelet-to-lymphocyte ratio (PLR) are simple and cost-effective inflammatory markers, well documented as indicators of postmenopausal osteoporosis. This study aimed to assess the relationships between these ratios and BMD in girls with AN.

Material and methods: The electronic records of 73 girls hospitalized for AN were analysed retrospectively. The age range of the study group was 12.56-17.67 years. BMD was assessed by dual-energy X-ray absorptiometry (DXA) and expressed as Z-scores according to lumbar spine (s-BMD) and total body (TB-BMD) sites. NLR and PLR were calculated according to complete blood count results. Patients were divided into 2 subgroups with parallel analyses - according to the TB-BMD criterion and the s-BMD criterion: normal (Z-score $>-2.0, \mathrm{n}=63$ ) and low s-BMD subgroup (Z-score $\leq-2.0, \mathrm{n}=10)$, and normal (Z-score $>-2.0, \mathrm{n}=45$ ) and low TB-BMD subgroup (Zscore $\leq-2.0, \mathrm{n}=28$ ).

Results: In the low s-BMD subgroup a tendency to an increase of mean NLR, PLR, and WBC values was observed. Respective BMD $Z$-score values correlated significantly and negatively with PLR in the low s-BMD $(R=-0.892, p<0.001)$ and normal TB-BMD $(R=-0.451$, $p=0.002)$ subgroups, while with NLR only in the normal TB-BMD subgroup $(R=-0.685, p<0.001)$. In the low s-BMD subgroup the PLR was shown to be a significant and independent predictor of s-BMD $(R=-0.881, p<0.001)$. The PLR contributed to $77.6 \%$ of the s-BMD $Z$-score variability $\left(R^{2}=0.776, p<0.001\right)$. In the normal TB-BMD subgroup, the PLR and NLR levels were significant and independent predictors of TB-BMD $(R=-0.352, p=0.004 ; R=-0.450, p=0.001 ; R=-0.339, p=0.005$, respectively $)$ and explained 44.4\% of TB-BMD Z-score variability $\left(\mathrm{R}^{2}=0.444, \mathrm{p}<0.001\right)$.

Conclusions: These results indicate that there might be a relationship between bone mass loss and inflammation expressed as NLR and PLR in adolescent girls suffering from AN. These connections seem to be dependent on the examined skeletal area. NLR and PLR, which are common indicators of morbidity and mortality in many malignancies and inflammatory chronic diseases, can also be useful in the evaluation of bone condition in adolescent females with AN. However, there is a need for further investigation in this field. (Endokrynol Pol 2021; 72 (4): 336-346)
\end{abstract}

Key words: anorexia nervosa; bone mineral density; neutrophil-to-lymphocyte ratio; platelet-to-lymphocyte ratio

\section{Introduction}

Anorexia nervosa (AN) is an eating disorder characterized by weight loss due to behaviours such as the limiting of food intake, the use of a low-calorie diet, excessive exercise, usage of laxatives, and self-induced vomiting
[1]. The incidence of AN is estimated at $0.3-2 \%$, and the ratio in women and men ranges between 10:1 and $4: 1[1,2]$.

AN affects the functioning of the whole body. It has been widely acknowledged that one of its most severe consequences is a decrease in bone mineral density 
(BMD), which is associated with a 7-fold increased risk of spontaneous fractures [3]. Decreased BMD has been reported in $85 \%$ of adult women with AN [4]. Its aetiology is multifactorial, and the mechanisms remain unclear. Inflammation is supposed to be one of the factors contributing to decreased BMD $[5,6]$. The latest data suggest that inflammation plays a critical role in bone remodelling and in the pathogenesis of postmenopausal osteoporosis [5]. It is now well established that the bones and the immune system are functionally connected $[7,8]$. According to some authors, proinflammatory cytokines may play a role in the mechanism that causes either a decrease or a complete lack of the expected increase in BMD [6, 9]. These cytokines (e.g. IL-1, IL-6, TNF- $\alpha$, IL-11, IL-15) directly and indirectly play a modulating role in bone remodelling. They do it via the receptor activator of nuclear factor- $\mathrm{B}$ ligand/receptor activator of nuclear factor- B/osteoprotegerin (RANKL/RANK/OPG) pathway. The RANKL-mediated signalling system plays here an essential role in the regulation and activation of osteoclastogenesis and osteoclastic bone resorption [7-10]. In addition, different immune cells such as macrophages, monocytes, B and T lymphocytes, mast cells, natural killer cells, etc. have been shown to influence skeletal status. These cells can enhance osteoclastogenesis in 2 ways [7]: 1) by increasing the production of bone-resorbing cytokines and 2) by increasing the numbers of precursors of osteoclasts.

According to the reported inflammatory background of postmenopausal osteoporosis in women, there is a relationship between the neutrophil-lymphocyte ratio (NLR), the platelet-lymphocyte ratio (PLR), and low BMD $[5,6,11,12]$.

It has been also suggested that NLR and PLR could be novel and independent osteopaenic markers in postmenopausal women $[5,10,11]$. These simple and cost-effective markers are well documented indicators of inflammation in various malignancies and inflammatory diseases $[5,6,10]$. Inflammatory cytokines, white blood cells, and platelets interact with each other and intensify the inflammatory response [13].

To the best of our knowledge, there are no studies that have established NLR and PLR norms for children. According to a study based on an adult healthy population aged 35-70 years (Tabari cohort, 10,255 participants), the normal values of the NLR and PLR are $1.70 \pm 0.70$ and $117.05 \pm 47.73$ [14]. However, these norms should not be considered as ranges of NLR and PLR in the paediatric population. Using adult NLR and PLR values in children is limited by age-related changes in the immune system. The qualitative and quantitative level of immune response changes according to age, especially in the group of adolescences. This point was confirmed by a study that compared complete blood count parameters and lymphocyte subsets in the group of infants, children, adolescents, adults, and the elderly. It was revealed that there are progressive declines in the percentage of total lymphocytes, absolute numbers of $\mathrm{T}$ and $\mathrm{B}$ cells, and pro-inflammatory cytokines according to age [15].

The method of choice in measuring the bone mass, which was introduced in 1980s, remains dual-energy X-ray absorptiometry (DXA). According to the current guidelines, low bone mineral density in children and adolescents is diagnosed when the Z-score in the DXA result is $\leq-2.0$ [16-19].

We hypothesized that in girls with AN there would be a correlation between NLR and PLR and skeletal status expressed as bone mineral density results. To test this hypothesis, we conducted a comparative analysis as well as correlation and regression analysis of NLR and PLR in girls suffering from AN and normal BMD compared with those in girls with AN and low BMD. To the best of our knowledge, this association has not been evaluated in adolescent patients with AN.

\section{Material and methods}

Electronic records of girls hospitalized for AN from 2015 to 2018 were analysed retrospectively. Participants with chronic disease known to affect bone metabolism or taking medications that influence skeletal status were excluded from the study. In total, 73 girls were included in data analyses. All examined patients were at IV-V Tanner puberty stage, they were in the acute phase of AN and were diagnosed with anorexia nervosa according to the DSM-5 diagnostic criteria for AN (20). The mean age was $15.14 \pm 1.34$ years (range 12.56-17.67), height $162.3 \pm 6.2 \mathrm{~cm}$, height SDS $-0.2 \pm 1.0 \mathrm{~cm}$, body mass $39.17 \pm 6.07 \mathrm{~kg}$, body mass SDS $-2.52 \pm 1.09 \mathrm{~kg}$, BMI $14.80 \pm$ $1.74 \mathrm{~kg} / \mathrm{m}^{2}$ BMI SDS $-2.95 \pm 0.98 \mathrm{~kg} / \mathrm{m}^{2}$, and the average duration of the disease was $13.49 \pm 10.86$ months. Sixty-seven girls were amenorrhoeic, and the mean duration of amenorrhoea was $7.80 \pm$ 6.42 months (4-28 months). Six girls were premenarchal. There were no patients with clinical manifestations indicating actual or recently acute infection or with a history of any fracture. Detailed information is given in Table 1 . The data used in this retrospective analysis were collected as part of a study that has been approved by the Ethics Committee.

\section{Anthropometric measurements}

Height $(\mathrm{cm})$ to the nearest $0.1 \mathrm{~cm}$ was measured using a single stadiometer, body mass $(\mathrm{kg})$ to the nearest $0.1 \mathrm{~kg}$ was assessed on electronic scale, and body mass index (BMI) was calculated as weight divided by squared height $\left(\mathrm{kg} / \mathrm{m}^{2}\right)$. Weight and BMI were expressed as absolute values and in the form of standard deviation score (SDS) [21].

\section{Bone mineral density}

Bone mineral density was assessed by dual-energy $X$-ay absorptiometry (DXA) in the first 2 weeks of hospital stay. Measured sites were: lumbar spine (s-BMD) and total body (TB-BMD) performed with a Hologic Explorer (USA). The results of BMD were expressed as Z-scores according to age and sex, calculated on the basis of the reference values. The instrument was calibrated with the manufacturer's recommendations. According to the TB-BMD criterion and the s-BMD criterion, patients were divided into 2 subgroups (s-BMD and TB-BMD) with parallel analyses used: normal — "within the 
Table 1. Clinical characteristics of girls with ano rexia nervosa (AN)

\begin{tabular}{|c|c|c|}
\hline \multirow{2}{*}{ Variables } & \multicolumn{2}{|c|}{ AN $(n=73)$} \\
\hline & Mean \pm SD & Range of values \\
\hline Age [year] & $15.14 \pm 1.34$ & $12.56-17.67$ \\
\hline Body mass $[\mathrm{kg}]$ & $39.17 \pm 6.07$ & $24.70-52.00$ \\
\hline Body mass SDS & $-2.52 \pm 1.09$ & $-0.5-(-5.68)$ \\
\hline Body mass loss $[\mathrm{kg}]$ & $14.52 \pm 9.01$ & $2.00-58.00$ \\
\hline Rate of body mass loss [kg/months] & $1.08 \pm 0.83$ & $0.75-1.50$ \\
\hline $\mathrm{BMI}\left[\mathrm{kg} / \mathrm{m}^{2}\right]$ & $14.80 \pm 1.74$ & $9.77-18.27$ \\
\hline BMI SDS & $-2.95 \pm 0.98$ & $-5.09-(-1.04)$ \\
\hline Disease duration [months] & $13.49 \pm 10.86$ & $3.00-51.00$ \\
\hline \multicolumn{3}{|l|}{ Menstrual status: } \\
\hline Amenorrhoea [months] & $7.80 \pm 6.42$ & $4.00-28.00$ \\
\hline Premenarchal [girls] & 6 & \\
\hline \multirow{2}{*}{$\begin{array}{l}\text { WBC }\left[10^{3} / \mu \mathrm{L}\right] \\
\text { Normal range } 4-10.5\left[10^{3} / \mu \mathrm{L}\right]\end{array}$} & Medians and quartiles & Range of values \\
\hline & $5.10(4.20-6.10)$ & $2.46-9.92$ \\
\hline $\begin{array}{l}\text { PLT }\left[10^{3} / \mu \mathrm{L}\right] \\
\text { Normal range } 150-450\left[10^{3} / \mu \mathrm{L}\right]\end{array}$ & $222.00(187.0-259.0)$ & $37.00-361.00$ \\
\hline $\begin{array}{l}\text { NEUT }\left[10^{3} / \mu \mathrm{L}\right] \\
\text { Normal range } 1.6-6\left[10^{3} / \mu \mathrm{L}\right]\end{array}$ & $2.42(1.65-3.24)$ & $0.66-6.95$ \\
\hline $\begin{array}{l}\text { LYMPH }\left[10^{3} / \mu \mathrm{L}\right] \\
\text { Normal range } 1.3-3.3\left[10^{3} / \mu \mathrm{L}\right]\end{array}$ & $1.85(1.85-2.69)$ & $1.09-3.49$ \\
\hline $\begin{array}{l}\text { MPV [fL] } \\
\text { Normal range } 6-9.5[\mathrm{Fl}]\end{array}$ & $8.90(8.20-9.50)$ & $6.90-12.20$ \\
\hline NLR & $1.10(0.79-1.49)$ & $0.40-4.48$ \\
\hline PLR & $102.50(81.75-124.77)$ & $14.45-244.62$ \\
\hline
\end{tabular}

Data expressed as mean \pm SD for normally distributed variables and as medians and quartiles for non-normally distributed variables; $S D$ - standard deviation; SDS — standard deviation score; BMI — body mass index; WBC — white blood cells; PLT — platelets; NEUT — neutrophils; LYMPH — lymphocytes; MPV — mean platelet volume; NLR — neutrophil-lymphocyte ratio; PLR — platelet-lymphocyte ratio

expected range for age" (Z-score $>-2.0, \mathrm{n}=63)$ and low - "below the expected for age" s-BMD subgroups (Z-score $\leq-2.0, \mathrm{n}=10)$, and normal $(\mathrm{Z}$-score $>-2.0, \mathrm{n}=45)$ and low TB-BMD subgroups (Z-score $\leq-2.0, \mathrm{n}=28)$, respectively.

\section{Biochemical analysis}

All blood samples were collected after a 12-h overnight fast between 8.00 and 9.00 a.m. on the first day of hospital stay. On the day of examination none of the girls presented symptoms of an acute infection. Complete blood count was performed at the hospital laboratory using Sysmex XN 1000 and Sysmex XN 350 analysers. NLR and PLR were calculated using the following formulas: neutrophil count/lymphocyte count and platelet count/lymphocyte count, respectively.

\section{Statistical analysis}

The database was prepared using Excel 2016 (Microsoft corporation). Statistical analysis was carried out with Statistica 13.3 for Windows (StatSoft Inc., USA). The normality of the distribution of the study sample was assessed using the Shapiro-Wilk test; homogeneity of variance was calculated using Leven's test. Data were expressed as mean \pm SD for normally distributed variables (clinical, anthropometric, and densitometric), and as medians and quartiles for non-normally distributed ones (WBC, PLT, NEUT, LYMPH, MPV, NLR, PLR). In the case of normal distribution of variables, the significance between examined subgroups of patients with normal and low s-BMD and TB-BMD were performed by
Student's t-test. In the case of non-normal distribution of variables, the significance was tested using the Mann-Whitney U-test. The distribution of blood count parameters was not normal and the number of participants (especially in s-BMD subgroup with Zscore $\leq-2.0$ ) was low, so Spearman's nonparametric correlation test was used to summarize the relationships between WBC, PLT, NEUT, LYMPH, MPV, NLR, PLR, and clinical, anthropometric, and densitometric measurements. The multivariate stepwise regression was used to determine the independent factors influencing BMD at various skeletal regions in the examined subgroups of girls with AN. Values for blood count parameters were linearized by logarithmic transformation when necessary. This analysis was performed in each subgroup of patients separately. The level of statistical significance was set at $\alpha \leq 0.05$. When more than 2 variables were analysed, Holma-Benferroni correction was evaluated.

\section{Results}

Baseline characteristics and complete blood count parameters in girls with AN are presented in Table 1. Baseline characteristics and complete blood count parameters in subgroups of patients according to the DXA result are shown in Table 2.

In low s- and TB-BMD subgroups the mean BMI SDS was significantly lower than in subgroups with 
Table 2. Clinical characteristics in subgroups of girls with anorexia nervosa and normal (Z-score $>-2.0)$ and low (Z-score $\leq-2.0)$ bone mineral density of lumbar spine (s-BMD) and bone mineral density of total body (TB-BMD)

\begin{tabular}{|c|c|c|c|c|c|c|}
\hline Variables & $\begin{array}{c}\text { s-BMD } \\
\text { Z-score }>-2.0 \\
n=63\end{array}$ & $\begin{array}{c}\text { s-BMD } \\
\text { Z-score } \leq-2.0 \\
n=10\end{array}$ & $\mathbf{p}$ & $\begin{array}{c}\text { TB-BMD } \\
\text { Z-score }>-2.0 \\
n=45\end{array}$ & $\begin{array}{c}\text { TB-BMD } \\
\text { Z-score } \leq-\mathbf{2 . 0} \\
n=28\end{array}$ & p \\
\hline Age [year] & $15.12 \pm 1.32$ & $15.30 \pm 1.49$ & 0.695 & $15.16 \pm 1.34$ & $15.05 \pm 1.36$ & 0.740 \\
\hline Body mass [kg] & $39.92 \pm 5.64$ & $34.42 \pm 6.81$ & $0.007^{*}$ & $39.90 \pm 5.52$ & $37.81 \pm 6.74$ & 0.157 \\
\hline Body mass SDS & $-2.38 \pm 1.00$ & $-3.40 \pm 0.90$ & $0.002^{*}$ & $-2.35 \pm 1.00$ & $-2.72 \pm 1.03$ & 0.153 \\
\hline Body mass loss [kg] & $9.67 \pm 6.59$ & $15.31 \pm 9.15$ & $0.020^{\wedge}$ & $10.96 \pm 5.51$ & $16.47 \pm 10.00$ & $0.003^{*}$ \\
\hline $\begin{array}{l}\text { Rate of body mass loss } \\
{[\mathrm{kg} / \text { months }]}\end{array}$ & $0.79 \pm 0.70$ & $0.70 \pm 0.57$ & 0.701 & $0.93 \pm 0.71$ & $1.01 \pm 0.70$ & 0.748 \\
\hline $\mathrm{BMI}\left[\mathrm{kg} / \mathrm{m}^{2}\right]$ & $14.99 \pm 1.60$ & $13.62 \pm 2.20$ & $0.020^{\wedge}$ & $14.89 \pm 1.54$ & $14.62 \pm 2.03$ & 0.638 \\
\hline BMI SDS & $-2.81 \pm 0.92$ & $-3.68 \pm 1.06$ & $0.008^{*}$ & $-2.80 \pm 0.95$ & $-3.55 \pm 1.04$ & $0.003^{*}$ \\
\hline Disease duration [months] & $12.27 \pm 9.48$ & $22.00 \pm 16.03$ & $0.009^{*}$ & $11.78 \pm 7.75$ & $16.33 \pm 14.30$ & 0.082 \\
\hline \multicolumn{7}{|l|}{ Menstrual status: } \\
\hline $\begin{array}{l}\text { Amenorrhoea } \\
\text { [months] }\end{array}$ & $7.38 \pm 6.14$ & $11.29 \pm 8.08$ & 0.078 & $7.69 \pm 6.12$ & $8.00 \pm 7.06$ & 0.843 \\
\hline $\begin{array}{l}\text { WBC }\left[10^{3} / \mu \mathrm{L}\right] \\
\text { Normal range } 4.0-10.5 \\
10^{3} / \mu \mathrm{L}\end{array}$ & $\begin{array}{c}5.05 \\
(4.20-5.95)\end{array}$ & $\begin{array}{c}5.63 \\
(3.65-7.40)\end{array}$ & $0.027^{\wedge}$ & $\begin{array}{c}5.10 \\
(4.20-6.10)\end{array}$ & $\begin{array}{c}5.20 \\
(4.05-6.40)\end{array}$ & 0.489 \\
\hline $\begin{array}{l}\text { PLT }\left[10^{3} / \mu \mathrm{L}\right] \\
\text { Normal range } 150-450 \\
10^{3} / \mu \mathrm{L}\end{array}$ & $\begin{array}{c}222.00 \\
(187.00-268.00)\end{array}$ & $\begin{array}{c}206.50 \\
(141.00-41.00)\end{array}$ & 0.051 & $\begin{array}{c}221.00 \\
(190,00-249.00)\end{array}$ & $\begin{array}{c}222.22 \\
(185.50-250.50)\end{array}$ & 0.424 \\
\hline $\begin{array}{l}\text { NEUT }\left[10^{3} / \mu \mathrm{L}\right] \\
\text { Normal range } 1.6-6.0 \\
10^{3} / \mu \mathrm{L}\end{array}$ & $\begin{array}{c}2.40 \\
(1.56-3.12)\end{array}$ & $\begin{array}{c}2.56 \\
(1.70-4.00)\end{array}$ & 0.351 & $\begin{array}{c}2.52 \\
(1.65-3.39)\end{array}$ & $\begin{array}{c}2.28 \\
(1.65-2.71)\end{array}$ & 0.404 \\
\hline $\begin{array}{l}\text { LYMPH }\left[10^{3} / \mu \mathrm{L}\right] \\
\text { Normal range } 1.3-3.3 \\
10^{3} / \mu \mathrm{L}\end{array}$ & $\begin{array}{c}2.16 \\
(1.86-2.71)\end{array}$ & $\begin{array}{c}2.07 \\
(1.62-2.33)\end{array}$ & 0.201 & $2.12(1.86-2.59)$ & $\begin{array}{c}2.20 \\
(1.70-2.82)\end{array}$ & 0.242 \\
\hline $\begin{array}{l}\text { MPV }[\mathrm{fL}] \\
\text { Normal range } 6-9.5 \mathrm{fl}\end{array}$ & $\begin{array}{c}9.00 \\
(8.10-9.60)\end{array}$ & $\begin{array}{c}8.67 \\
(7.70-8.90)\end{array}$ & 0.330 & $\begin{array}{c}9.00 \\
(8.20-9.60)\end{array}$ & $\begin{array}{c}8.55 \\
(7.80-9.20)\end{array}$ & 0.108 \\
\hline NLR & $\begin{array}{c}1.10 \\
(0.71-1.44)\end{array}$ & $\begin{array}{c}1.42 \\
(1.05-1.88)\end{array}$ & $0.037^{\wedge}$ & $\begin{array}{c}1.20 \\
(0.82-1.54)\end{array}$ & $\begin{array}{c}0.95 \\
(0.74-1.25)\end{array}$ & 0.307 \\
\hline PLR & $\begin{array}{c}102.12 \\
(81.67-125.33)\end{array}$ & $\begin{array}{c}116.85 \\
(95.86-124.77)\end{array}$ & $0.047^{\wedge}$ & $\begin{array}{c}103.76 \\
(81.75-126.40)\end{array}$ & $\begin{array}{c}97.72 \\
(78.75-118.64)\end{array}$ & 0.267 \\
\hline
\end{tabular}

Data expressed as mean \pm SD for normally distributed variables and as medians and quartiles for not normally distributed variables. Z-score - the number of SD from age-matched subjects; SD — standard deviation; BMI — body mass index; SDS — standard deviation score; WBC — white blood cells; PLT — platelets;

NEUT — neutrophils; LYMPH — lymphocytes; MPV — mean platelet volume; NLR — neutrophil-lymphocyte ratio; PLR — platelet-lymphocyte ratio;

${ }^{*}$ Differences reach the $\alpha$ level of significance $(<0.05)$ adjusted by the Holma-Bonferroni correction; ^ ^ Differences do not reach the $\alpha$ level of significance $(<0.05)$ adjusted by the Holma-Bonferroni correction

normal s-BMD, and the mean duration of the disease was significantly longer in the low compared to the normal s-BMD subgroup. Moreover, in the low s-BMD subgroup, a tendency towards an increase in mean NLR, PLR, and WBC values was observed.

Table 3 shows correlation between values of blood count parameters and clinical and selected anthropometric parameters in subgroups of patients, according to the DXA result. There are given relationships only between these parameters, which correlated significantly in at least one subgroup of patients with AN. In the normal TB-BMD subgroup, PLR correlated sig- nificantly and positively with amenorrhoea $(R=0.466$, $p=0.001$ ). In the low TB-BMD subgroup, NLR and PLR correlated significantly and negatively with the rate of body mass loss $(\mathrm{R}=-0.552$, $\mathrm{p}=0.006$ and $\mathrm{R}=-0.562$, $p=0.002$, respectively) and the duration of the disease $(\mathrm{R}=-0.523, \mathrm{p}=0.006$ and $\mathrm{R}=-0.573, \mathrm{p}=0.001$, respectively). In the case of remaining blood count values and clinical and anthropometric parameters, there was also a tendency towards an increase of $R$ values in selected parameters. Detailed information is given in Table 3.

Tables 4 and 5 show correlations between respective s- and TB-BMD Z-score values and examined blood 
Table 3. Correlation between values of white blood cells (WBC), platelets (PLT), neutrophils (NEUT), lymphocytes (LYMPH), mean platelet volume (MPV), neutrophil-lymphocyte ratio (NLR) and platelet-lymphocyte ratio (PLR), and clinical and selected anthropometric parameters in subgroups of girls with AN and normal (Z-score $>-2.0)$ and low (Z-score $\leq-2.0)$ bone mineral density of lumbar spine (s-BMD) and bone mineral density of total body (TB-BMD)

\begin{tabular}{|c|c|c|c|c|c|c|c|c|c|}
\hline \multirow[t]{2}{*}{ Variables } & & \multicolumn{2}{|c|}{$\begin{array}{c}\text { s-BMD } \\
\text { Z-score }>-2.0 \\
n=63\end{array}$} & \multicolumn{2}{|c|}{$\begin{array}{c}\text { s-BMD } \\
\text { Z-score } \leq-2.0 \\
n=10 \\
\end{array}$} & \multicolumn{2}{|c|}{$\begin{array}{c}\text { TB-BMD } \\
\text { Z-score }>-2.0 \\
n=45 \\
\end{array}$} & \multicolumn{2}{|c|}{$\begin{array}{c}\text { TB-MD } \\
\text { Z-score } \leq-2.0 \\
n=28\end{array}$} \\
\hline & & $\mathbf{R}$ & $\mathbf{p}$ & $\mathbf{R}$ & p & $\mathbf{R}$ & p & $\mathbf{R}$ & $\mathbf{p}$ \\
\hline WBC $\left[10^{3} / \mu \mathrm{L}\right]$ & \multirow{7}{*}{ Age [years] } & -0.190 & 0.136 & -0.224 & 0.533 & -0.093 & 0.541 & -0.280 & 0.149 \\
\hline $\operatorname{PLT}\left[10^{3} / \mu \mathrm{L}\right]$ & & -0.214 & 0.091 & -0.091 & 0.802 & -0.168 & 0.271 & -0.231 & 0.237 \\
\hline NEUT $\left[10^{3} / \mu \mathrm{L}\right]$ & & 0.001 & 0.992 & 0.007 & 0.987 & 0.061 & 0.692 & -0.126 & 0.521 \\
\hline 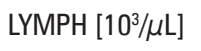 & & -0.366 & $0.003^{*}$ & -0.624 & 0.053 & -0.299 & $0.046^{\wedge}$ & -0.535 & $0.003 *$ \\
\hline MPV [fL] & & 0.169 & 0.186 & 0.717 & $0.020^{\wedge}$ & 0.139 & 0.363 & 0.262 & 0.178 \\
\hline NLR & & -0.121 & 0.343 & -0.284 & 0.425 & -0.162 & 0.288 & -0.125 & 0.528 \\
\hline PLR & & -0.030 & 0.814 & 0.030 & 0.934 & -0.076 & 0.621 & 0.014 & 0.944 \\
\hline WBC $\left[10^{3} / \mu \mathrm{L}\right]$ & \multirow{7}{*}{ Body mass [kg] } & -0.192 & 0.132 & 0.079 & 0.829 & -0.259 & 0.086 & 0.045 & 0.822 \\
\hline PLT $\left[10^{3} / \mu \mathrm{L}\right]$ & & -0.150 & 0.241 & -0.067 & 0.854 & -0.264 & 0.079 & 0.050 & 0.800 \\
\hline NEUT $\left[10^{3} / \mu \mathrm{L}\right]$ & & -0.059 & 0.646 & 0.231 & 0.521 & -0.178 & 0.242 & 0.129 & 0.514 \\
\hline LYMPH $\left[10^{3} / \mu \mathrm{L}\right]$ & & -0.284 & $0.024^{\wedge}$ & -0.261 & 0.467 & -0.330 & $0.027^{\wedge}$ & -0.108 & 0.583 \\
\hline MPV [fL] & & 0.321 & $0.010^{\wedge}$ & 0.328 & 0.354 & 0.497 & $<0.001^{*}$ & 0.095 & 0.630 \\
\hline NLR & & -0.014 & 0.915 & -0.164 & 0.651 & 0.107 & 0.486 & -0.100 & 0.612 \\
\hline PLR & & 0.054 & 0.676 & -0.115 & 0.751 & 0.102 & 0.503 & -0.084 & 0.671 \\
\hline WBC $\left[10^{3} / \mu \mathrm{L}\right]$ & \multirow{7}{*}{$\begin{array}{l}\text { Rate of body } \\
\text { mass loss } \\
\text { [kg/months] }\end{array}$} & 0.119 & 0.358 & -0.183 & 0.637 & 0.022 & 0.886 & 0.051 & 0.804 \\
\hline $\operatorname{PLT}\left[10^{3} / \mu \mathrm{L}\right]$ & & -0.229 & 0.074 & -0.267 & 0.488 & -0.293 & 0.051 & -0.090 & 0.663 \\
\hline NEUT $\left[10^{3} / \mu \mathrm{L}\right]$ & & -0.064 & 0.623 & -0.335 & 0.379 & -0.068 & 0.654 & -0.243 & 0.230 \\
\hline LYMPH $\left[10^{3} / \mu \mathrm{L}\right]$ & & 0.304 & $0.016^{\wedge}$ & 0.200 & 0.606 & 0.221 & 0.144 & 0.405 & $0.040^{\wedge}$ \\
\hline MPV [fL] & & 0.228 & 0.074 & -0.638 & $0.042^{\wedge}$ & 0.315 & 0.035 & -0.118 & 0.567 \\
\hline NLR & & 0.230 & 0.072 & 0.067 & 0.865 & 0.206 & 0.174 & -0.522 & $0.006^{*}$ \\
\hline PLR & & -0.005 & 0.967 & 0.100 & 0.798 & -0.025 & 0.871 & -0.562 & $0.002^{*}$ \\
\hline WBC $\left[10^{3} / \mu \mathrm{L}\right]$ & \multirow{7}{*}{ BMI [kg/m²] } & -0.122 & 0.341 & 0.188 & 0.603 & -0.187 & 0.218 & 0.118 & 0.551 \\
\hline PLT $\left[10^{3} / \mu \mathrm{L}\right]$ & & -0.064 & 0.619 & -0.006 & 0.987 & -0.146 & 0.339 & 0.041 & 0.835 \\
\hline NEUT $\left[10^{3} / \mu \mathrm{L}\right]$ & & -0.070 & 0.584 & 0.353 & 0.317 & -0.157 & 0.303 & 0.164 & 0.405 \\
\hline 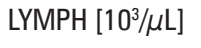 & & -0.183 & 0.151 & -0.139 & 0.701 & -0.278 & 0.064 & -0.013 & 0.946 \\
\hline MPV [fL] & & 0.189 & 0.138 & 0.316 & 0.374 & 0.475 & $<0.001^{*}$ & -0.056 & 0.777 \\
\hline NLR & & 0.054 & 0.676 & -0.188 & 0.603 & 0.143 & 0.350 & -0.065 & 0.742 \\
\hline PLR & & 0.089 & 0.490 & -0.212 & 0.556 & 0.129 & 0.398 & -0.083 & 0.676 \\
\hline WBC $\left[10^{3} / \mu \mathrm{L}\right]$ & \multirow{7}{*}{$\begin{array}{l}\text { Disease duration } \\
\text { [months] }\end{array}$} & -0.170 & 0.183 & 0.067 & 0.864 & -0.058 & 0.703 & -0.174 & 0.387 \\
\hline PLT $\left[10^{3} / \mu \mathrm{L}\right]$ & & 0.139 & 0.278 & 0.350 & 0.356 & 0.263 & 0.081 & -0.064 & 0.750 \\
\hline NEUT $\left[10^{3} / \mu \mathrm{L}\right]$ & & 0.024 & 0.851 & 0.084 & 0.831 & 0.003 & 0.982 & 0.125 & 0.535 \\
\hline LYMPH $\left[10^{3} / \mu \mathrm{L}\right]$ & & -0.298 & $0.018^{\wedge}$ & -0.267 & 0.488 & -0.127 & 0.404 & -0.528 & $0.005^{*}$ \\
\hline MPV [fL] & & -0.246 & 0.052 & 0.717 & $0.020^{\wedge}$ & -0.286 & 0.057 & 0.060 & 0.765 \\
\hline NLR & & -0.199 & 0.119 & 0.167 & 0.668 & -0.120 & 0.431 & -0.523 & $0.006^{*}$ \\
\hline PLR & & 0.026 & 0.839 & 0.117 & 0.765 & 0.107 & 0.486 & -0.573 & $0.001^{*}$ \\
\hline WBC $\left[10^{3} / \mu \mathrm{L}\right]$ & \multirow{7}{*}{$\begin{array}{l}\text { Amenorrhoea } \\
\text { [months] }\end{array}$} & -0.217 & 0.102 & 0.464 & 0.177 & -0.209 & 0.184 & 0.012 & 0.957 \\
\hline PLT $\left[10^{3} / \mu \mathrm{L}\right]$ & & 0.099 & 0.461 & 0.357 & 0.431 & 0.250 & 0.110 & -0.139 & 0.527 \\
\hline NEUT $\left[10^{3} / \mu \mathrm{L}\right]$ & & -0.047 & 0.726 & 0.357 & 0.431 & -0.085 & 0.594 & 0.064 & 0.773 \\
\hline LYMPH $\left[10^{3} / \mu \mathrm{L}\right]$ & & -0.356 & $0.006^{*}$ & 0.179 & 0.702 & -0.347 & $0.024^{\wedge}$ & -0.219 & 0.315 \\
\hline MPV [fL] & & -0.257 & 0.051 & -0.487 & 0.268 & -0.361 & $0.019^{\wedge}$ & -0.009 & 0.968 \\
\hline NLR & & -0.133 & 0.319 & 0.071 & 0.879 & -0.103 & 0.515 & -0.129 & 0.557 \\
\hline PLR & & 0.054 & 0.686 & 0.143 & 0.760 & 0.466 & $0.001^{*}$ & -0.134 & 0.542 \\
\hline
\end{tabular}

Z-score - the number of SD from age-matched subjects; SD — standard deviation; SDS — standard deviation score; *Correlation reach the $\alpha$ level of significance $(<0.05)$ adjusted by the Holma-Bonferroni correction; ^ Correlation do not reach the $\alpha$ level of significance $(<0.05)$ adjusted by the Holma-Bonferroni correction 
Table 4. Correlation between respective values of bone mineral density of lumbar spine (s-BMD) expressed as Z-score with white blood cells (WBC), platelets (PLT), neutrophils (NEUT), lymphocytes (LYMPH), mean platelet volume (MPV), neutrophillymphocyte ratio (NLR), and platelet-lymphocyte ratio (PLR) in the group of girls with anorexia nervosa (AN) as a whole and in subgroups of patients with normal Z-score (>-2.0) and low Z-score $(\leq-2.0)(s-B M D)$

\begin{tabular}{|c|c|c|c|c|c|c|}
\hline \multirow{3}{*}{ Variables } & \multirow{2}{*}{\multicolumn{2}{|c|}{$\begin{array}{c}\text { Girls with AN (whole group) } \\
\begin{array}{c}\text { s-BMD Z-score } \\
n=73\end{array} \\
\end{array}$}} & \multicolumn{4}{|c|}{ Subgroups of girls with AN } \\
\hline & & & \multicolumn{2}{|c|}{$\begin{array}{c}\text { s-BMD } \\
\text { Z-score }>-2.0 \mathrm{n}=63\end{array}$} & \multicolumn{2}{|c|}{$\begin{array}{c}\text { s-BMD } \\
\text { Z-score } \leq-2.0 \mathrm{n}=10\end{array}$} \\
\hline & $\mathbf{R}$ & $\mathbf{p}$ & $\mathbf{R}$ & $\mathbf{p}$ & $\mathbf{R}$ & $\mathbf{p}$ \\
\hline WBC $\left[10^{3} / \mu \mathrm{L}\right]$ & -0.070 & 0.555 & -0.058 & 0.653 & 0.012 & 0.973 \\
\hline $\mathrm{PLT}\left[10^{3} / \mu \mathrm{L}\right]$ & 0.060 & 0.616 & 0.042 & 0.746 & -0.123 & 0.735 \\
\hline NEUT $\left[10^{3} / \mu \mathrm{L}\right]$ & -0.086 & 0.470 & -0.022 & 0.864 & -0.148 & 0.683 \\
\hline LYMPH $\left[10^{3} / \mu \mathrm{L}\right]$ & 0.040 & 0.739 & -0.051 & 0.689 & 0.449 & 0.193 \\
\hline MPV [fL] & 0.017 & 0.887 & -0.038 & 0.768 & 0.111 & 0.760 \\
\hline NLR & -0.087 & 0.462 & -0.015 & 0.906 & 0.209 & 0.568 \\
\hline PLR & -0.236 & $0.044^{\wedge}$ & 0.105 & 0.413 & -0.892 & $<0.001 *$ \\
\hline
\end{tabular}

Z-score - the number of SD from age-matched subjects; R — Spearman's correlation coefficient; ${ }^{*}$ Correlation reached the $\alpha$ level of significance (< 0.05$)$ adjusted by the Holma-Bonferroni correction; ^ ${ }^{\wedge}$ Correlation do not reach the $\alpha$ level of significance $(<0.05)$ adjusted by the Holma-Bonferroni correction

Table 5. Correlation between values of bone mineral density of total body (TB-BMD) expressed as Z-score with white blood cells (WBC), platelets (PLT), neutrophils (NEUT), lymphocytes (LYMPH), mean platelet volume (MPV), neutrophil-lymphocyte ratio (NLR), and platelet-lymphocyte ratio (PLR) in the group of girls with anorexia nervosa (AN) and subgroups of patients with normal (Z-score $>-2.0)$ and low (Z-score $\leq-2.0)$ TB-BMD

\begin{tabular}{|c|c|c|c|c|c|c|}
\hline \multirow{3}{*}{ Variables } & \multirow{2}{*}{\multicolumn{2}{|c|}{$\begin{array}{c}\text { Girls with AN (whole group) } \\
\begin{array}{c}\text { TB-BMD Z-score } \\
n=73\end{array}\end{array}$}} & \multicolumn{4}{|c|}{ Subgroups of girls with AN } \\
\hline & & & \multicolumn{2}{|c|}{$\begin{array}{c}\text { TB-BMD } \\
\text { Z-score }>-2.0 \mathrm{n}=45\end{array}$} & \multicolumn{2}{|c|}{$\begin{array}{c}\text { TB-BMD } \\
\text { Z-score } \leq-2.0 \mathrm{n}=\mathbf{2 8}\end{array}$} \\
\hline & $\mathbf{R}$ & $\mathbf{p}$ & $\mathbf{R}$ & $\mathbf{p}$ & $\mathbf{R}$ & $\mathbf{p}$ \\
\hline WBC $\left[10^{3} / \mu \mathrm{L}\right]$ & -0.037 & 0.752 & -0.154 & 0.313 & 0.093 & 0.638 \\
\hline $\mathrm{PLT}\left[10^{3} / \mu \mathrm{L}\right]$ & 0.033 & 0.782 & -0.065 & 0.670 & 0.264 & 0.175 \\
\hline NEUT $\left[10^{3} / \mu \mathrm{L}\right]$ & 0.012 & 0.922 & -0.235 & 0.120 & 0.146 & 0.459 \\
\hline LYMPH $\left[10^{3} / \mu \mathrm{L}\right]$ & -0.062 & 0.602 & -0.004 & 0.980 & 0.058 & 0.769 \\
\hline MPV [fL] & 0.023 & 0.848 & 0.254 & 0.092 & -0.092 & 0.643 \\
\hline NLR & -0.239 & $0.042^{\wedge}$ & -0.685 & $<0.001^{*}$ & -0.136 & 0.851 \\
\hline PLR & -0.231 & $0.049^{\wedge}$ & -0.451 & $0.002^{*}$ & -0.037 & 0.490 \\
\hline
\end{tabular}

${ }^{*}$ Correlation reach the $\alpha$ level of significance $(<0.05)$ adjusted by the Holma-Bonferroni correction; ^ Correlation did not reach the $\alpha$ level of significance $(<0.05)$ adjusted by the Holma-Bonferroni correction

count parameters in the whole group of girls with AN and in subgroups divided according to the DXA result. In the whole group of girls suffering from AN only a tendency towards negative correlation between s-BMD Z-score and PLR was observed $(\mathrm{R}=-0.236, \mathrm{p}=0.044)$. The correlation did not reach the a level of significance adjusted by the Holma-Bonferroni correction. In the normal s-BMD subgroup no correlation was observed between $>-2.0$ s-BMD Z-score values and examined blood count parameters ( $p>0.050$ ). In the low s-BMD subgroup a significant and negative correlation was observed only between $\leq-2.0$ s-BMD Z-score values and PLR $(\mathrm{R}=-0.892, \mathrm{p}<0.001)$ (Tab. 4).

In the whole group of patients with AN only a tendency towards a negative correlation between
TB-BMD Z-score values and NLR and PLR was observed $(R=-0.239, p=0.042 ; R=-0.231, p=0.049$, respectively). Correlation do not reach the a level of significance adjusted by the Holma-Bonferroni correction. In the normal TB-BMD subgroup a significant and negative correlation was observed between $>-2.0$ TB-BMD Z-score values and NLR $(\mathrm{R}=-0.685, \mathrm{p}<0.001)$ and PLR $(R=-0.451, p=0.002)$. In the low TB-BMD subgroup no significant correlation was observed between $\leq-2.0$ TB-BMD Z-score values and examined blood count parameters ( $p>0.050)$ (Tab. 5).

Using a stepwise regression analysis, we examined how much of the variance in BMD at various skeletal regions could be explained by selected blood count parameters in girls with AN as a whole and in sub- 
Table 6. Stepwise regression modelling for predictors of changes in bone mineral density of lumbar spine (s-BMD) expressed as Z-score in girls with anorexia nervosa $(A N)$ as a whole and subgroups of patients with normal (Z-score $>-2.0)$ and low (Z-score $\leq-2.0)$ s-BMD

\begin{tabular}{|c|c|c|c|c|c|c|c|c|c|}
\hline \multirow{4}{*}{$\begin{array}{l}\text { Independent } \\
\text { variables }\end{array}$} & \multirow{2}{*}{\multicolumn{3}{|c|}{ Girls with AN (whole group) }} & \multicolumn{6}{|c|}{ Subgroups of girls with AN } \\
\hline & & & & \multicolumn{3}{|c|}{ Normal } & \multicolumn{3}{|c|}{ Low } \\
\hline & \multicolumn{3}{|c|}{$\begin{array}{c}\text { s-BMD Z-score } \\
\text { as a dependent variable }\end{array}$} & \multicolumn{3}{|c|}{$\begin{array}{c}\text { s-BMD Z-score }>-2.0 \\
\text { as a dependent variable }\end{array}$} & \multicolumn{3}{|c|}{$\begin{array}{c}\text { s-BMD Z-score }>-2.0 \\
\text { as a dependent variable }\end{array}$} \\
\hline & $\mathbf{R}$ & $\mathbf{R}^{2} \mathbf{i}$ & $\mathbf{p}$ & $\mathbf{R}$ & $\mathbf{R}^{2} \mathbf{i}$ & $\mathbf{p}$ & $\mathbf{R}$ & $\mathbf{R}^{2} \mathbf{i}$ & p \\
\hline MPV [fL] & - & - & - & - & - & - & - & - & - \\
\hline NLR & - & - & - & - & - & - & - & - & - \\
\hline \multirow[t]{2}{*}{ PLR } & -0.201 & 0.040 & 0.088 & - & - & - & -0.881 & 77.62 & $<0.001$ \\
\hline & & $\begin{array}{l}e \mathrm{R}=0 \\
.04014 \\
=0.088\end{array}$ & & \multicolumn{3}{|c|}{ Multiple $\mathrm{R}=0.0$} & \multicolumn{3}{|c|}{$\begin{array}{c}\text { Multiple } R=0.881 \\
\text { Multiple } \mathrm{R}^{2}=0.776(77.62 \%) \\
\qquad \mathrm{p}<0.001\end{array}$} \\
\hline
\end{tabular}

Z-score; the number of the SD from age-matched subjects; PLR — platelet-lymphocyte ratio; NLR — neutrophil-lymphocyte ratio; MPV mean platelet volume; $\mathrm{R}$ - Spearman's correlation coefficients; $\mathrm{R}^{2}$ - multiple determination coefficient; $\mathrm{R}^{2} \mathrm{i}$ - individual determination coefficient

Table 7. Stepwise regression modelling for predictors of changes in bone mineral density of total body (TB-BMD) expressed as Z-score in girls with anorexia nervosa $(A N)$ as a whole and in subgroups of patients with normal (Z-score $>-2.0)$ and low (Z-score $\leq-2.0)$ TB-BMD

\begin{tabular}{|c|c|c|c|c|c|c|c|c|c|}
\hline \multirow{4}{*}{$\begin{array}{l}\text { Independent } \\
\text { variables }\end{array}$} & \multirow{2}{*}{\multicolumn{3}{|c|}{ Girls with AN (whole group) }} & \multicolumn{6}{|c|}{ Subgroups of girls with AN } \\
\hline & & & & \multicolumn{3}{|c|}{ Normal } & \multicolumn{3}{|c|}{ Low } \\
\hline & \multicolumn{3}{|c|}{$\begin{array}{c}\text { TB-BMD Z-score } \\
\text { as a dependent variable }\end{array}$} & \multicolumn{3}{|c|}{$\begin{array}{l}\text { TB-BMD Z-score }>-2.0 \\
\text { as a dependent variable }\end{array}$} & \multicolumn{3}{|c|}{$\begin{array}{l}\text { TB-BMD Z-score } \leq-2.0 \\
\text { as a dependent variable }\end{array}$} \\
\hline & $\mathbf{R}$ & $\mathbf{R}^{2} \mathbf{i} \%$ & $\mathbf{p}$ & $\mathbf{R}$ & $\mathbf{R}^{2} \mathbf{i} \%$ & $\mathbf{p}$ & $\mathbf{R}$ & $\mathbf{R}^{2} \mathbf{i} \%$ & $\mathbf{p}$ \\
\hline MPV [fL] & - & - & - & 0.339 & 11.79 & 0.005 & - & - & - \\
\hline NLR & -0.324 & 10.50 & 0.030 & -0.450 & 20.25 & 0.001 & - & - & - \\
\hline \multirow[t]{2}{*}{ PLR } & -0.159 & 2.53 & 0.277 & -0.352 & 12.39 & 0.004 & - & - & - \\
\hline & \multicolumn{3}{|c|}{$\begin{aligned} \text { Multiple } R=0.361 \\
\text { Multiple } \mathrm{R}^{2}=0.130(13.03 \%) \\
\mathrm{p}=0.062\end{aligned}$} & \multicolumn{3}{|c|}{$\begin{array}{c}\text { Multiple } R=0.666 \\
\text { Multiple } \mathrm{R}^{2}=0.444(44.36 \%) \\
\mathrm{p}<0.001\end{array}$} & \multicolumn{3}{|c|}{ Multiple $\mathrm{R}=0.0$} \\
\hline
\end{tabular}

Z-score; the number of the SD from age-matched subjects; PLR — platelet-lymphocyte ratio; NLR — neutrophil-lymphocyte ratio; MPV mean platelet volume; $\mathrm{R}$ - Spearman's correlation coefficients; $R^{2}$ - multiple determination coefficient; $R^{2} i$ - individual determination coefficient

groups of patients, according to the DXA result. The covariates entered into the models were MPV, NLR, and/or PLR. Other blood count parameters (WBC, PLT, NEUT, LYMPH) were not introduced into the model due to collinearity.

Tables 6 and 7 show the values of standardized regression $\mathrm{R}$ coefficients, which allows a comparison of relative contributions of incorporating independent variable into stepwise regression models of respective s- and TB-BMD Z-scores as dependent variables.

In the whole group of girls with AN only PLR was entered into the model with s-BMD as a dependent variable, but PLR is not a significant independent predictor of s-BMD ( $p>0.05)$. In the normal s-BMD subgroup none of the independent variables was entered into the model with $>-2.0$ s-BMD Z-score as a dependent variable. In the low s-BMD subgroup PLR was shown to be a significant independent predictor $(R=-0.881$, $\mathrm{p}<0.001)$ in the model with $\leq-2.0 \mathrm{~s}$-BMD Z-score as a dependent variable. PLR contributed to $77.62 \%$ of low s-BMD variability (Multiple $\mathrm{R}^{2}=0.776, \mathrm{p}<0.001$ ) (Tab. 6).

In the whole group of patients with AN, NLR and PLR were entered into the model with TB-BMD as a dependent variable, but only NLR was shown to be a significant independent predictor $(R=-0.324$, $\mathrm{p}=0.030)$ in this model. NLR contributed to $10.50 \%$ of TB-BMD variability. In normal TB-BMD subgroup MPV, NLR and PLR values were significant and independent predictors $(\mathrm{R}=0.339, \mathrm{p}=0.005 ; \mathrm{R}=-0.450$, $\mathrm{p}=0.001 ; \mathrm{R}=-0.352, \mathrm{p}=0.004$, respectively) in the model with $>-2.0$ TB-BMD Z-score as a dependent variable. Taken together, MPV, NLR, and PLR values contributed to $44.36 \%$ of normal TB-BMD Z-score vari- 
ability (multiple $\mathrm{R}^{2}=0.444, \mathrm{p}<0.001$ ). MPV contributed to $11.79 \%$, NLR contributed to $20.25 \%$, while PLR contributed to $12.39 \%$ of normal TB-BMD variability. In the low TB-BMD subgroup none of the independent variables was entered into the model of $\leq-2.0 \mathrm{~TB}$-BMD Z-score as a dependent variable.

The presented correlation and regression analysis data indicate that comparative correlation and regression analysis with the division of the whole study group into subgroups with normal and low BMD results are more detailed and accurate, and also more interesting from the clinical point of view than the analysis of the entire study group.

\section{Discussion}

The most relevant findings of this study include associations of NLR and PLR with BMD Z-scores. These connections seem to be dependent on the examined skeletal area, because the PLR was shown to be a significant and independent predictor of s-BMD and TB-BMD, and the NLR was a significant and independent predictor of TB-BMD.

The adolescent years are a critical period for bone mineral accrual to achieve an optimal peak bone mass (PBM), which is an important determinant of fracture risk in adult life. It is estimated that more than $90 \%$ of PBM is achieved by the end of the second decade [22]. Any skeletal deterioration that occurs during this period may affect bone health later in adult life. The adolescent years are also the time when the onset of AN occurs. One of the consequences of AN is a decrease in BMD, or lack of an adequate bone mass accrual [22]. Rapid bone loss, which occurs within 6 months of disease onset and persists despite weight recovery, is well documented in adult women with AN. The prognosis is worse when disease begins during puberty [3, 22-24]. The aetiology of low BMD in AN is multifactorial and includes hypogonadism, undernutrition, low levels of IGF-1, hypercortisolaemia, excessive exercise, and resistance to growth hormone [22]. However, taking into account the relationship between immunity and bone, it seems that an inflammatory background could be an important factor leading to a BMD decrease in girls suffering from AN. Evidence suggests that inflammation plays a critical role in bone metabolism and in the pathogenesis of osteoporosis. The inflammatory background of postmenopausal osteoporosis is well known. It was reported that C-reactive protein - a sensitive systemic inflammatory marker - is positively correlated with bone loss in healthy pre- and postmenopausal women [25]. Moreover, osteoporosis is more common in conditions with chronic inflammation such as rheumatoid arthritis (RA) and systemic lupus erythematosus, haematological diseases, inflammatory bowel disease, chronic obstructive pulmonary disease, etc. [10]. Additionally, hypoestrogenism leads to up-regulation of pro-inflammatory cytokines, which contribute to the activation of osteoclasts [10]. Elevated levels of inflammatory cytokines that have been linked with lower BMD in postmenopausal women were also reported [25]. The mechanisms by which chronic inflammatory factors modulate bone resorption are known [26, 27]: 1 - pro-inflammatory cytokines have a final common mediator of osteoclast function: RANK and RANKL, and indirectly act on mesenchymal stem cells and osteoclast precursors to increase osteoclast-mediated bone resorption; 2 - osteoclastogenesis can be modified through the modulation of macrophage colony stimulating factor.

In adolescent girls with AN the alterations of the immune system and elevated levels of selected pro-inflammatory cytokines are well documented. Misra et al. showed that levels of the high-sensitivity C-reactive protein were decreased, while levels of IL-6 increased in these patients [28]. Other investigators found also elevated concentrations of TNF- $\alpha$ [29]. In the study of Dalton et al. a range of inflammatory markers in patients with AN was measured. They found that IL-6, IL-15, and vascular cell adhesion molecule (VCAM-1) were significantly elevated, compared with health controls. Two meta-analyses of cytokines in AN showed significantly higher concentrations of IL-6, IL1-R, and TNF-a, and decreased C-reactive protein level [30, 31]. Correlations between pro-inflammatory cytokines, bone metabolism markers, and RANKL/RANK/OPG signalling system cytokines indicate that pro-inflammatory cytokines may be involved in the bone loss mechanisms. This is most likely due to the impact on the RANKL/RANK/OPG pathway [9], where RANKL is considered to be the key osteoclastogenic cytokine [27]. In the course of rheumatoid arthritis, it was observed that several pro-inflammatory cytokines upregulated RANKL on synovial fibroblasts. The elevated expression of RANKL activated osteoclastogenesis and resulted in severe bone destruction [32]. Moreover, T lymphocytes were proven to express RANKL and promote bone loss in inflammatory arthritis [33].

NLR and PLR were reported to be closely related to systemic inflammation and immune response status [34]. Recent data showed that raised NLR and/or PLR were associated with i.a. mortality in acute pulmonary embolism [35], severity of coronary heart disease, short-term and long-term mortalities in patients presenting with non-ST elevation myocardial infarction [36], critical limb ischaemia in peripheral arterial occlusive disease [37], or mortality in patients with acute coronary syndrome [38]. Fisher et al. found that NLR was a significant risk factor and a moderate predictor 
of poorer postoperative outcomes such as myocardial injury, high inflammatory response/infection, and in-hospital death [39]. Due to the fact that osteoporosis can be induced and maintained by chronic inflammation, as well as the fact that NLR and PLR have been known as new markers of the systemic inflammatory activation, it seems that these ratios should be useful as markers of osteoporosis.

The results of our study showed a tendency towards increased mean NLR values in low s-BMD adolescent females with AN, and NLR turned out to be an independent predictor of TB-BMD in the $>-2.0 \mathrm{Z}$-score subgroup. These results are consistent with reports concerning postmenopausal women; however, there are no studies concerning NLR in osteoporotic adolescent girls. Huang [5], Yu [40], and Yilmaz [10] observed that the NLR ratio was higher in postmenopausal women with osteoporosis. In a study concerning elderly men and women, Öztürk observed that the NLR ratio was significantly higher in people with osteoporosis [12]. All of these authors found that the NLR ratio could be a significant risk factor for postmenopausal osteoporosis. In a study of postmenopausal Korean women, it was shown that NLR was negatively associated with the mean lumbar BMD [26]. In a report of a large group of postmenopausal women, Fang et al. observed that NLR was an independent risk factor for postmenopausal osteoporosis [34]. Moreover, several studies have shown that an elevated NLR was associated with poor prognosis in osteoporotic individuals [5, 27]. It was also proven that an elevated NLR was an independent indicator of fracture in orthogeriatric patients [39] and that it increased the risk of fracture [34]. In turn, the studies of Eroglu and Koseoglu [6, 11] showed no significant correlation between NLR and BMD.

The study of Poubelle et al. [33] explains the role of neutrophils and lymphocytes in local bone remodelling. The authors observed the expression of the membrane-associated form of RANKL in healthy blood neutrophils and the expression of the membrane-associated form of RANKL, RANK, and secretion of OPG in inflammatory neutrophils from patients with RA. In the context of RANKL/RANK interactions between cells, including osteoclasts, these findings suggest that neutrophils could play a dual role as immune and bone-like cells during the inflammatory process [33]. Moreover, Chakravarti et al. observed that lipopolysaccharide up-regulates the expression of membrane RANKL in human blood neutrophils, and through RANKL neutrophils it activates osteoclasts, resulting in bone loss [41]. In another study Riegel et al. [42] stated that polymorphonuclear neutrophils upon stimulation express RANK in vivo and in vitro and migrate toward RANKL. In light of these reports, it is possible that neutrophils contribute to the pathogenesis of osteoporosis. This may be due to the enhanced expression of RANKL and osteoclasts activation. Neutrophils could also act directly through RANK.

In addition, our study showed a tendency towards an increase in mean PLR, NLR, and WBC values in the low s-BMD subgroup. Moreover, respective s-BMD Zscore values correlated significantly and negatively with PLR in the low s-BMD and normal TB-BMD subgroups, while with NLR this was seen in the normal TB-BMD subgroup. PLR also transpired to be a significant and independent predictor of low s-BMD and normal TB-BMD, while NLR and MPV proved to be significant and independent predictors of normal TB-BMD. These results indicate that the sensitivity of different examined skeletal areas to the effects of the studied blood count parameters may be different in patients with AN. That could be related to age, degree of malnutrition, duration of the disease, or amenorrhoea $(17,43,44)$. This is indicated not only by the obtained results of the regression analysis, but also by the results of the correlation analysis. In the normal s-BMD subgroup of AN patients the lymphocyte count correlated significantly and negatively with age and amenorrhoea. In the normal TB-BMD subgroup MPV correlated significantly and positively with body mass and BMI, while PLR correlated significantly and positively with amenorrhoea. However, in the low TB-BMD subgroup the lymphocyte count correlated significantly and negatively with age and the duration of the disease, while NLR and PLR correlated significantly and negatively with the rate of body mass loss and the duration of the disease.

Consistent with our report, in a study of postmenopausal women, Eroglu and Koseoglu observed significantly higher PLR values in the low BMD patients [6, 11]. Additionally, Koseoglu found PLR to be a discriminative factor for low BMD [6]. In turn, in the study of San-Hui et al. [26] there was no significant relationship between PLR and BMD. It is thought that platelets contribute to postmenopausal osteoporosis because the interaction of some factors in platelets with vitamin $\mathrm{D}$ receptors is important for bone turnover [11]. Another conception is that PLT can contribute to inflammation by releasing thromboxane [11].

Interesting results were reported by Eroglu et al. [45] - in a study of women with postpartum osteoporosis they found that NLR and PLR were lower in the low BMD group, but the differences were not significant. These results suggest that inflammation is not a key factor in every case of osteoporosis.

Our research shows different DXA results, according to the different examined skeletal areas. When discussing the results, it should be considered whether the relationships found for s- and TB-BMD are equally 
important from the clinical point of view. It seems that the connections regarding s-BMD could be more important: the difference between the groups with normal and low BMD and statistically significant dependencies within the subgroup with low s-BMD. For TB-BMD, no differences were found between subjects with normal and low BMD, and the correlation and regression analyses revealed significant relationships only in the subgroup with normal DXA results. It was found that the uneven impact on BMD disorders is related to the age of AN onset. An onset of AN at a younger age mostly affects the development or maintenance of cortical bone [43]. This observation is based on the fact that before puberty appendicular growth is more rapid than axial, whereas during puberty appendicular growth slows and axial growth accelerates [43]. Therefore, the differences in the DXA results can be caused by the fact that the posteroanterior lumbar spine and the total body less head sites reflect the trabecular and cortical bone, respectively [17, 44].

It is possible that the observed differences are due to the stronger impact of inflammation on trabecular bone. This thesis seems to be confirmed by studies conducted among patients suffering from chronic joint inflammatory diseases. In patients with RA a global decrease of BMD was seen in more than 50\% of cases. The decrease in BMD occurs most often in the forearm. However, these results do not correlate with the femoral neck- and s-BMD. This may be due to the fact that the severity of the inflammation is greatest in the wrist. The next location where bone loss occurs most often is the femoral neck. A BMD decrease is observed there six times more often than in the control group. However, there was no difference in s-BMD in patients with RA and in the control group [46]. In the study comparing patients with rheumatoid arthritis and psoriatic arthritis (PsA), lumbar spine-BMD was higher in PsA patients. However, there were no differences in femoral neck BMD. A significant correlation between the duration of the disease and femoral neck BMD in both groups was also observed. Relationship between disease duration and s-BMD was noted only in RA patients [47].

A limitation of this study is lack of a control group, but parents of healthy girls did not agree for a DXA examination because of radiation.

In conclusion, our study indicates that there might be a relationship between low bone mineral density in adolescent girls suffering from AN and inflammation expressed as NLR and PLR. It seems that these connections may be dependent on the examined skeletal area. However, to develop a full picture of the impact of inflammatory on bone health in adolescent girls with $\mathrm{AN}$, there is a need for further investigations in this field.

\section{References}

1. Malczyk Ż, Oświęcimska JM. Gastrointestinal complications and refeeding guidelines in patients with anorexia nervosa. Psychiatr Pol. 2017 51(2): 219-229, doi: 10.12740/PP/65274, indexed in Pubmed: 28581533.

2. Smink FRE, van Hoeken D, Hoek HW. Epidemiology of eating disorders: incidence, prevalence and mortality rates. Curr Psychiatry Rep. 2012; 14(4): 406-414, doi: 10.1007/s11920-012-0282-y, indexed in Pubmed: 22644309.

3. Oświecimska J, Ziora K, Pluskiewicz W, et al. Skeletal status and laboratory investigations in adolescent girls with anorexia nervosa. Bone. 2007; 41(1): 103-110, doi: 10.1016/j.bone.2007.03.018, indexed in Pubmed: 17493887.

4. Shepherd S, Kyriakou A, Shaikh MG, et al. Longitudinal changes in bone parameters in young girls with anorexia nervosa. Bone. 2018; 116: 22-27, doi: 10.1016/j.bone.2018.03.022, indexed in Pubmed: 29601899.

5. Huang C, Li S. Association of blood neutrophil lymphocyte ratio in the patients with postmenopausal osteoporosis. Pak J Med Sci. 2016; 32(3): 762-765, doi: 10.12669/pjms.323.10292, indexed in Pubmed: 27375729 .

6. Koseoglu SB, Koseoglu SB. Bone loss \& platelet-to-lymphocyte ratio. Biomark Med. 2017; 11(1): 5-10, doi: 10.2217/bmm-2016-0188, indexed in Pubmed: 27917655.

7. Zupan J, Jeras M, Marc J, et al. Osteoimmunology and the influence of pro-inflammatory cytokines on osteoclasts. Biochem Med (Zagreb). 2013 23(1): 43-63, doi: 10.11613/bm.2013.007, indexed in Pubmed: 23457765.

8. Majewski PMa. Osteoimmunologia, czyli o wzajemnych oddziaływaniach komórek układów kostnego i odpornościowego. Kosmos. 2017; 66(317): 665-75.

9. Ostrowska Z, Ziora K, Oświęcimska J, et al. Selected pro-inflammatory cytokines, bone metabolism, osteoprotegerin, and receptor activator of nuclear factor-kB ligand in girls with anorexia nervosa. Endokrynol Pol. 2015; 66(4): 313-321, doi: 10.5603/EP.2015.0040, indexed in Pubmed: 26323468

10. Yilmaz H, Uyfun M, Yilmaz TS, et al. Neutrophil-lymphocyte ratio may be superior to C-reactive protein for predicting the occurrence of postmenopausal osteoporosis. Endocr Regul. 2014; 48(1): 25-33, doi: 10.4149/endo_2014_01_25, indexed in Pubmed: 24524373.

11. Eroglu S, Karatas G, Eroglu S, et al. Platelet/lymphocyte ratio is an independent predictor for osteoporosis. Saudi Med J. 2019; 40(4): 360-366, doi: 10.15537/smj.2019.4.24009, indexed in Pubmed: 30957129.

12. Öztürk ZA, Yesil Y, Kuyumcu ME, et al. Inverse relationship between neutrophil lymphocyte ratio (NLR) and bone mineral density (BMD) in elderly people. Arch Gerontol Geriatr. 2013; 57(1): 81-85, doi: 10.1016/j. archger.2013.02.005, indexed in Pubmed: 23490023.

13. Czerwiec K, Myślińska D, Wądołowska A, et al. Charakterystyka mediatorów zapalenia - rola cytokin prozapalnych. Ann Acad Medicae Gedanensis. 2016; 46(1): 53-8.

14. Moosazadeh M, Maleki I, Alizadeh-Navaei R, et al. Normal values of neutrophil-to-lymphocyte ratio, lymphocyte-to-monocyte ratio and platelet-to-lymphocyte ratio among Iranian population: Results of Tabari cohort. Caspian J Intern Med. 2019; 10(3): 320-325, doi: 10.22088/cjim.10.3.320, indexed in Pubmed: 31558995

15. Valiathan R, Ashman M, Asthana D, et al. Effects of Ageing on the Immune System: Infants to Elderly. Scand J Immunol. 2016; 83(4): 255-266, doi: 10.1111/sji.12413, indexed in Pubmed: 26808160.

16. Bachrach LK, Gordon CM, Bachrach LK, et al. Bone Densitometry in Children and Adolescents. Pediatrics. 2016; 138(4), doi: 10.1542/peds.2016-2398, indexed in Pubmed: 27669735.

17. Crabtree NJ, Arabi A, Bachrach LK, et al. International Society for Clinical Densitometry, International Society for Clinical Densitometry. Dual-energy $\mathrm{X}$-ray absorptiometry interpretation and reporting in children and adolescents: the revised 2013 ISCD Pediatric Official Positions. J Clin Densitom. 2014; 17(2): 225-242, doi: 10.1016/j.jocd.2014.01.003, indexed in Pubmed: 24690232

18. Gordon CM, Leonard MB, Zemel BS, et al. International Society for Clinical Densitometry, International Society for Clinical Densitometry. 2013 Pediatric Position Development Conference: executive summary and reflections. J Clin Densitom. 2014; 17(2): 219-224, doi: 10.1016/j. jocd.2014.01.007, indexed in Pubmed: 24657108.

19. Morawiecka-Pietrzak M, Ziora K, Ostrowska Z. Interpretation of bone densitometry and definition of osteoporosis in children and adolescents. Pediatr Pol. 2019; 94(4): 241-247, doi: 10.5114/polp.2019.88041.

20. American Psychiatric Association. Diagnostic and Statistical Manual of Mental Disorders (DSM-5) 2013. Diagnostic and Statistical Manual of Mental Disorders (DSM-5

21. Palczewska I, Niedźwiedzka Z. Wskaźniki rozwoju somatycznego u dzieci i młodzieży warszawskiej. Med Wieku Rozw . 2001; 5 (Suppl. 1): $1-112$

22. Misra M, Klibanski A. Anorexia nervosa and osteoporosis. Rev Endocr Metab Disord. 2006; 7(1-2): 91-99, doi: 10.1007/s11154-006-9005-1, indexed in Pubmed: 16972186. 
23. Mumford J, Kohn M, Briody J, et al. Long-term Outcomes of Adolescent Anorexia Nervosa on Bone. J Adolesc Health. 2019; 64(3): 305-310, doi: 10.1016/j.jadohealth.2018.07.025, indexed in Pubmed: 30819332

24. Misra M, Klibanski A. Anorexia nervosa and osteoporosis. Rev Endocrine Metab Dis. 2006; 7(1-2): 91-99, doi: 10.1007/s11154-006-9005-1.

25. Ilesanmi-Oyelere BL, Schollum L, Kuhn-Sherlock B, et al. Inflammatory markers and bone health in postmenopausal women: a cross-sectional overview. Immun Ageing. 2019; 16: 15, doi: 10.1186/s12979-019-0155-x, indexed in Pubmed: 31333751.

26. Lee SH, Ryu SY, Park J, et al. The Relationship of Neutrophil-Lymphocyte Ratio and Platelet-Lymphocyte Ratio with Bone Mineral Density in Korean Postmenopausal Women. Chonnam Med J. 2019; 55(3): 150-155, doi: 10.4068/cmj.2019.55.3.150, indexed in Pubmed: 31598472.

27. Lacativa PG, Farias ML. Osteoporosis and inflammation. Arq Bras Endocrinol Metabol. 2010; 54(2): 123-132, doi: 10.1590/s0004-27302010000200007, indexed in Pubmed: 20485900.

28. Misra M, Miller KK, Tsai $\mathrm{P}$, et al. Uncoupling of cardiovascular risk markers in adolescent girls with anorexia nervosa. J Pediatr. 2006; 149(6): 763-769, doi: 10.1016/j.jpeds.2006.08.043, indexed in Pubmed: 17137889.

29. Dalton B, Campbell IC, Chung R, et al. Inflammatory Markers in Anorexia Nervosa: An Exploratory Study. Nutrients. 2018; 10(11), doi: 10.3390/nu10111573, indexed in Pubmed: 30355978.

30. Dalton B, Bartholdy S, Robinson L, et al. A meta-analysis of cytokine concentrations in eating disorders. J Psychiatr Res. 2018; 103: 252-264, doi: 10.1016/j.jpsychires.2018.06.002, indexed in Pubmed: 29906710.

31. Solmi M, Veronese N, Favaro A, et al. Inflammatory cytokines and anorexia nervosa: A meta-analysis of cross-sectional and longitudinal studies. Psychoneuroendocrinology. 2015; 51: 237-252, doi: 10.1016/j. psyneuen.2014.09.031, indexed in Pubmed: 25462897.

32. Terashima A, Takayanagi H. Overview of Osteoimmunology. Calcif Tissue Int. 2018; 102(5): 503-511, doi: 10.1007/s00223-018-0417-1, indexed in Pubmed: 29589061.

33. Poubelle PE, Chakravarti A, Fernandes MJ, et al. Differential expression of RANK, RANK-L, and osteoprotegerin by synovial fluid neutrophils from patients with rheumatoid arthritis and by healthy human blood neutrophils. Arthritis Res Ther. 2007; 9(2): R25, doi: 10.1186/ar2137, indexed in Pubmed: 17341304.

34. Fang $\mathrm{H}$, Zhang $\mathrm{H}$, Wang Z, et al. Systemic immune-inflammation index acts as a novel diagnostic biomarker for postmenopausal osteoporosis and could predict the risk of osteoporotic fracture. J Clin Lab Anal. 2020; 34(1): e23016, doi: 10.1002/jcla.23016, indexed in Pubmed: 31423643.

35. Phan T, Brailovsky Y, Fareed J, et al. Neutrophil-to-Lymphocyte and Platelet-to-Lymphocyte Ratios Predict All-Cause Mortality in Acute Pulmonary Embolism. Clin Appl Thromb Hemost. 2020; 26:
1076029619900549, doi: 10.1177/1076029619900549, indexed in Pubmed: 31960711

36. Awan MS, Daud MY, Khan M, et al. Usefulness Of Neutrophils To Lymphocytes Ratio For Predicting Troponin-I Elevation In Patients Presenting With Suspected Nste-Acute Coronary Syndrome. J Ayub Med Coll Abbottabad. 2019; 31(Suppl 1)(4): S674-S677, indexed in Pubmed: 31965774.

37. Gary T, Pichler M, Belaj K, et al. Platelet-to-lymphocyte ratio: a novel marker for critical limb ischemia in peripheral arterial occlusive disease patients. PLoS One. 2013; 8(7): e67688, doi: 10.1371/journal.pone.0067688, indexed in Pubmed: 23844064.

38. Tamhane UU, Aneja S, Montgomery D, et al. Association between admission neutrophil to lymphocyte ratio and outcomes in patients with acute coronary syndrome. Am J Cardiol. 2008; 102(6): 653-657, doi: 10.1016/j. amjcard.2008.05.006, indexed in Pubmed: 18773982.

39. Fisher A, Srikusalanukul W, Fisher L, et al. The Neutrophil to Lymphocyte Ratio on Admission and Short-Term Outcomes in Orthogeriatric Patients. Int J Med Sci. 2016; 13(8): 588-602, doi: 10.7150/ijms.15445, indexed in Pubmed: 27499691.

40. Yu Xy, Li Xs, Li Y, et al. Neutrophil-lymphocyte ratio is associated with arterial stiffness in postmenopausal women with osteoporosis. Arch Gerontol Geriatr. 2015; 61(1): 76-80, doi: 10.1016/j.archger.2015.03.011, indexed in Pubmed: 25882272

41. Chakravarti A, Raquil MA, Tessier P, et al. Surface RANKL of Toll-like receptor 4-stimulated human neutrophils activates osteoclastic bone resorption. Blood. 2009; 114(8): 1633-1644, doi: 10.1182/blood-2008-09-178301, indexed in Pubmed: 19546479.

42. Riegel A, Maurer T, Prior B, et al. Human polymorphonuclear neutrophils express RANK and are activated by its ligand, RANKL. Eur J Immunol. 2012; 42(4): 975-981, doi: 10.1002/eji.201141786, indexed in Pubmed: 22531921.

43. Milos G, Spindler A, Rüegsegger P, et al. Cortical and trabecular bone density and structure in anorexia nervosa. Osteoporos Int. 2005; 16(7): 783-790, doi: 10.1007/s00198-004-1759-2, indexed in Pubmed: 15452690.

44. Estrada A, Ramnitz MS, Gafni RI. Bone densitometry in children and adolescents. Curr Opin Obstet Gynecol. 2014; 26(5): 339-346, doi: 10.1097/GCO.0000000000000100, indexed in Pubmed: 25144595.

45. Eroglu S, Karatas G, Aziz V, et al. Evaluation of bone mineral density and its associated factors in postpartum women. Taiwan J Obstet Gynecol. 2019; 58(6): 801-804, doi: 10.1016/j.tjog.2019.09.013, indexed in Pubmed: 31759531.

46. Bachta A, Kulig M, Tłustochowicz W. Osteoporoza w przewlekłych zapaleniach stawów. Post Nauk Med. 2012; 3: 218-225.

47. Spieler W, Schottstedt K, Keitel W, et al. Comparison of bone mineral density and fracture rates in rheumatoid arthritis and psoriatic arthritis. Osteopor Int. 1996; 6(S1): 289-289, doi: 10.1007/bf02500692. 